\title{
A new methodology to automatically segment biomedical images
}

\author{
A. Garrido, N. Pérez De La Blanca, M. García-Silvente \\ Departamento de Ciencias de la Computación e I.A. \\ ETS Ingeniería Informática \\ Universidad de Granada. 18071 Granada. Spain.
}

\begin{abstract}
This paper presents a new approach for 2D object segmentations using an automatic method applied on images with severe noise conditions and locating objects with a very high degree of deformation. We use a physically-based shape model to obtain a deformable template, which is defined on a canonical ortogonal coordinate system. The proposed methodology works from a set of samples and from the output of an edge detector to segment the objects by using a reformulated Hough transform (automatic initialization) together with an optimization procedure (on a learned surface of deformation). Results from biomedical images are presented.
\end{abstract}

Key Words : Deformable template, modal analysis, Hough transform, physically based modeling, finite element method.

\section{Introduction}

The problem of automatic detection, segmentation, and extraction of objects from a given scene is one of the most challenging paradigms in image analysis, taking into account that it is very important to obtain global solutions for all kinds of images. The methods that usually appear in the literature require the initial image to present particular conditions to be successful (well defined features, adequate and uniform illumination, absence of noise, non-overlapping objects,...). In some cases, it is very difficult or impossible to improve the imagecapturing process, so we need methods to segment object from images obtained in conditions very far from optimal.

We address the problem of locating objects in images that present mainly two problematic aspects: noise and deformations. Biomedical images present very interesting cases related to problems such as: absence of contrast, many objects in a single scene, low quality, etc. Furthermore, the deformations are a very important problem in biological objects. If we do not assume a contour model, contour extraction in the presence of noise, clutter and occlusion is an ill-posed problem [9]. Therefore, in this paper we propose a supervised method, based on a deformable template model, to detect and extract shapes. The object model that we propose is defined from a closed polygonal approximation of the contour 
and a coordinate system (learned from a set of samples) to handle deformations. To implement it, it is necessary to align the shapes ([10]), then, to process the obtained deformations in order to obtain the desired deformation axes. Once we have this object model, we can use it to initialize and optimize a deformable template.

In this paper, we study the main components of the proposed methodology. In section 2, we discuss the method to process the deformations and to obtain an object model. In sections 3 and 4 , we give the algorithms to initialize and optimize the position of the deformable template, respectively. Finally, in sections 5 and 6 , we present experimental results from some examples and conclusions.

\section{Deformation model}

Let us consider a shape (with null deformation) described by a vector

$$
X=\left(x_{1}, \cdots, x_{n}, y_{1}, \cdots, y_{n}\right)^{t}
$$

where $\left(x_{i}, y_{i}\right)$ are the nodal points along the contour (i.e., may be thought of as a polygonal approximation of the shape). Then, a deformed shape may be calculated from

$$
X_{d}=\mathrm{X}+\Phi v
$$

where $v$ is a $2 n \times 1$ vector and $\Phi$ is a $2 n \times 2 n$ matrix, that is, composed by $2 n$ vectors $\phi_{1} \cdots \phi_{2 n}$ each of them corresponding to an axis of deformation. So, the problem is to determine a good set of axes. We use a deformation system based on modal analysis, used to simulating the dynamic behavior of an object $([2])$ and proposed by Pentland and Sclaroff to align shapes ([8], [10]). In modal analysis, the standard FEM computations are simplified by posing the dynamic equations in terms of the equations' eigenvectors. The eigenmodes (known as the object's free vibration or deformation modes) of this physical shape are used to obtain a canonical, frequency-ordered orthogonal coordinate system. Moreover, these coordinate system allow us to separate rigid modes (displacements and rotations) from non-rigid modes and may be used to align a set of samples in order to process the deformations.

\subsection{Finite Element Method}

The mathematical formulation of the proposed model is based on the standard engineering technique for simulating the dynamic behavior of an object([2]). To use FEM, the object is represented from a set of nodal points as the finite element nodes. Then, stiffness and mass matrices are built and shape functions are used to relate the displacement (or others properties) of a single point to the relative displacements of all the other nodes of an object, so these interpolation functions allow continuous material properties. The polynomial shape function for each element may be written in vector form as: 


$$
u(x, y)=H(x, y) U
$$

where $(x, y)$ is the point where we want to know the displacement, $H$ is the interpolation matrix and $U$ is the vector composed by the displacements of each element node. To solve the problem of deforming an elastic body requires solving the dynamic equilibrium equation

$$
M \ddot{U}+D \dot{U}+K U=R
$$

where $R$ is the load vector, and $M, D, K$ are the mass, damping, and stiffness matrices, respectively.

\subsection{Modal analysis}

A technique to improve the solution of equations (4) is the change of basis to modal generalized displacements, that is, to transform the equilibrium equations into a more effective form for direct integration. The FEM governing equations can be decoupled by posing the equations in a basis defined by the $M$ orthogonalized eigenvectors of $K$. These eigenvectors (with respective eigenvalues) are the solution to the generalized eigenproblem:

$$
K \phi=w^{2} M \phi
$$

which will determine an optimal transformation basis set. There are $2 n$ eigensolutions

$$
\left(w_{1}^{2}, \phi_{1}\right),\left(w_{2}^{2}, \phi_{2}\right) \cdots\left(w_{2 n}^{2}, \phi_{2 n}\right)
$$

where all the eigenvectors are $M$-orthonormalized, i.e.

$$
\phi_{i}^{t} M \phi_{j}=\left\{\begin{array}{l}
1 \text { Si } i=j \\
0 \text { Si } i \neq j
\end{array}\right.
$$

and $0 \leq w_{1}^{2} \leq \cdots \leq w_{2 n}^{2}$

The eigenvector $\phi_{i}$ is called the $i$ th mode's shape vector and $w_{i}$ is the corresponding frequency of vibration. These vectors establish a very good basis to represent the deformations ([10]), separating the rigid body modes (displacements and rotations) from the non-rigid modes and providing a global-to-local ordering of shape deformations.

\subsection{Principal components analysis}

Once the deformations have been expressed using the free vibration modes, we have to select the principal components in order to get a coordinate system as simple as possible. Directly, we are able to select the main components from this basis, that is, we can take out the last modes (the highest frequency modes), which correspond to local shape variations (the ones most sensitive to noise) 
and so, we avoid the excessive number of dimensions caused by an oversampled contour. In spite of this, an easier way to select the principal components may be used, i.e. via the Karhunen-Loeve transform.

Let us suppose a set of samples $E_{i}(1 \leq i \leq m)$ corresponding to $m$ deformations from a template $T$ (obviously, of null deformation). The process of calculating principal components of this set of samples is:

To align each shape with the template $T$, processing both the rigid transformation and non-rigid deformation to obtain $m$ aligned samples $X_{i}$ (A very efficient method based on modal analysis is described in [10]). Once we have got the aligned samples, each of them with $n$ nodal points (see eq. (1)), we are able to calculate the $m$ vectors $U_{i}$ as

$$
U_{i}=X_{i}-T
$$

then, the transformation to modal coordinates is

$$
\tilde{U}_{i}=\Phi^{-1} U_{i}=\Phi^{t} M U_{i}
$$

where $\tilde{U}_{i_{1}}, \tilde{U}_{i_{2}}$ y $\tilde{U}_{i_{3}}$ are null because of the previous alignment. Thus, we are able to apply principal component analysis on the set of vectors:

$$
\tilde{V}_{i}=\left(\tilde{U}_{i_{4}}, \cdots, \tilde{U}_{i_{2 n}}\right)^{t}
$$

\section{Initialization}

In the literature we can find many works using deformable models but not an automatic initialization stage taking into account a very high degree of deformation, bearing in mind the overlapping objects or the generality of the solution, that is, to be able to apply the solutions to others applications. In this section we address the problem of initializing deformable templates of known objects in images with problems like many objects in a single scene, overlapping objects, partial information and noise.

Let us suppose the curve $C(t)=\left(f_{x}(t), f_{y}(t)\right)$ where $0 \leq t \leq L$ and

$$
\int_{0}^{L} f_{x}(t) d t=\int_{0}^{L} f_{y}(t) d t=0
$$

Let us suppose also a set of deformation axes

$$
D=\left\{e_{x}, e_{y}, e_{r}, e_{d_{1}}, \cdots, e_{d_{n}}\right\}
$$

where $\left(e_{x}, e_{y}, e_{r}\right)$ are the axes of translation and rotation. The next axes correspond to non-rigid deformations. Then the set of possible location of the curve $C$ is determined by $(3+n)$-tuples. Thus, our goal is to find a set of parameters defining the location of the objects, that is, to obtain a tuple that determines the object location

$$
\left(\triangle x, \triangle y, \triangle r, \triangle d_{1}, \cdots, \Delta d_{n}\right)
$$


Because of the kind of problems in our images, a powerful technique to use is the Hough transform ([1], [6]). The Hough transform uses a parameter space of dimension $3+n$, sampling each axis to build a discrete parameter space $E$ where the evidences in the images will be accumulated. The location with the highest level of evidences will be obtained looking for the maximun in this accumulator. However, it is impossible to directly apply it because, on the one hand, edges may be displaced due to a smoothing operation, to noise or to local deformations. The calculated position to update the accumulator will be not correct. On the other hand, the algorithm needs a lot of time and space to perform the location. The parameter space size is too large, so too much memory is needed to store the accumulator. In addition, each detected evidence in the image will need a lot of time (we will have to update a subset of positions in the accumulator).

Thus, we have to reformulate the GHT. The solution that we propose is:

- The results from GHT may be erroneous because the input information (for example, point along the contour from an edge detector) is very noisy. A solution is to use more stable information, i.e. a higher semantic level or several kinds of information (points, corners, segments, regions, etc). In this work straight segments have been used.

- If we want to use a method like GHT, we have to decrease the parameter space size. We propose that the method sacrifices accurate solution (i.e., accurate location), in order to be able to apply the reformulated GHT.

We reduce the accumulator size by selecting some axes (the dimension of the parameter space is reduced). In this sense, the coordinate system proposed in previous section allows us to take out a set of axes corresponding to local deformations, that is, we can select principal axes. Moreover, the selected axes are able to be sampled (the size of the parameter space is reduced). The result is a dictionary of shapes. Of course, it is possible to find an object in the image which is not included in the dictionary but there will be a similar contour, that is, an approximation. Using this reduced parameter space, the Hough transform does not work because the detected evidence votes in a position which may be displaced. To solve it, the parameter space is updated not only on a single position but on a uncertainty region (the set of positions where the location may be displaced due to local deformations). The result from this algorithm is only an approximation of the solution, however. this problem will be solved considering an optimization stage.

\section{Optimization}

Once we have an approximation of the solution, we have to optimize it. This stage may be accomplished by using a proper energy function and a proper algorithm to obtain the optimum. This global energy function is composed by an external energy function (which contributes with information from the image) and an internal energy function (which contributes with information from the 
object model). In order to design a robust method, we have used the learned surface of deformation, on which the functions have been defined.

To define the internal energy function, we have selected the simplest method; that is, we have delimited the admissible space of deformations from the learning samples (using the axes calculated from the given principal component analisys), although we can use more complex methods to include other a priori knowledge. On the other hand, the external energy function has been defined as follows:

Let us suppose the curve $C$ and the image $G(x, y)$. We are able to use a function $P(x, y, \theta)$ in order to define the following expression

$$
E_{e x t}(C)=\frac{1}{L} \int_{0}^{L} P\left(f_{x}(s), f_{y}(s), \theta(s)\right) d s
$$

where $\theta(t)$ is defined as the angle between the vectors

$$
\nabla G\left(f_{x}(t), f_{y}(t)\right) \quad \text { and } \quad\left(-\frac{d f_{y}}{d s}(t), \frac{d f_{x}}{d s}(t)\right)
$$

thus, it allows to use both, magnitude and direction of the gradient. The selected function $P$ has been defined as the distance to the nearest edgepoint (with gradiente direction in according to the curve direction). The learned surface of deformation together with this energy function produces a robust method to optimize the initial location of the solution. Therefore, we can use a simple optimization algorithm (steepest descent method has been used).

\section{Experimental results}

In this section we present the results on several images. We have considered two types of images: nematodes and hands. The uncertainty rcgion is a circle (radius $=10$ pixels). The paremeter space sizes (non-rigid deformations) have been reduced to 4 non-rigid axes for nematodes (sampling 7, 3,3,3 respectively) and 3 non-rigid axes for hands (sampling 5, 4,3 respectively).

Moreover, the rotation axis has been reduced to 36 , that is, the result is equivalent to add an axis with size 18.9 and 6 (respectively) to handle non-rigid deformations. In figure 2 we present the final results after optimization (results from the hands that we have not drawn are similar).

\section{Conclusions}

In this paper we have described a new approach for $2 \mathrm{D}$ object segmentations using an automatic method applied on images with problems as partial information, overlapping objects, many objects in a single scene, severe noise conditions and locating objects with a very high degree of deformation. We have illustrated the problem of segmenting images using different examples.

In this paper, that the Hough transform may be used to fit the initial position of a template has been demostrated by reformulating a new algorithm, 


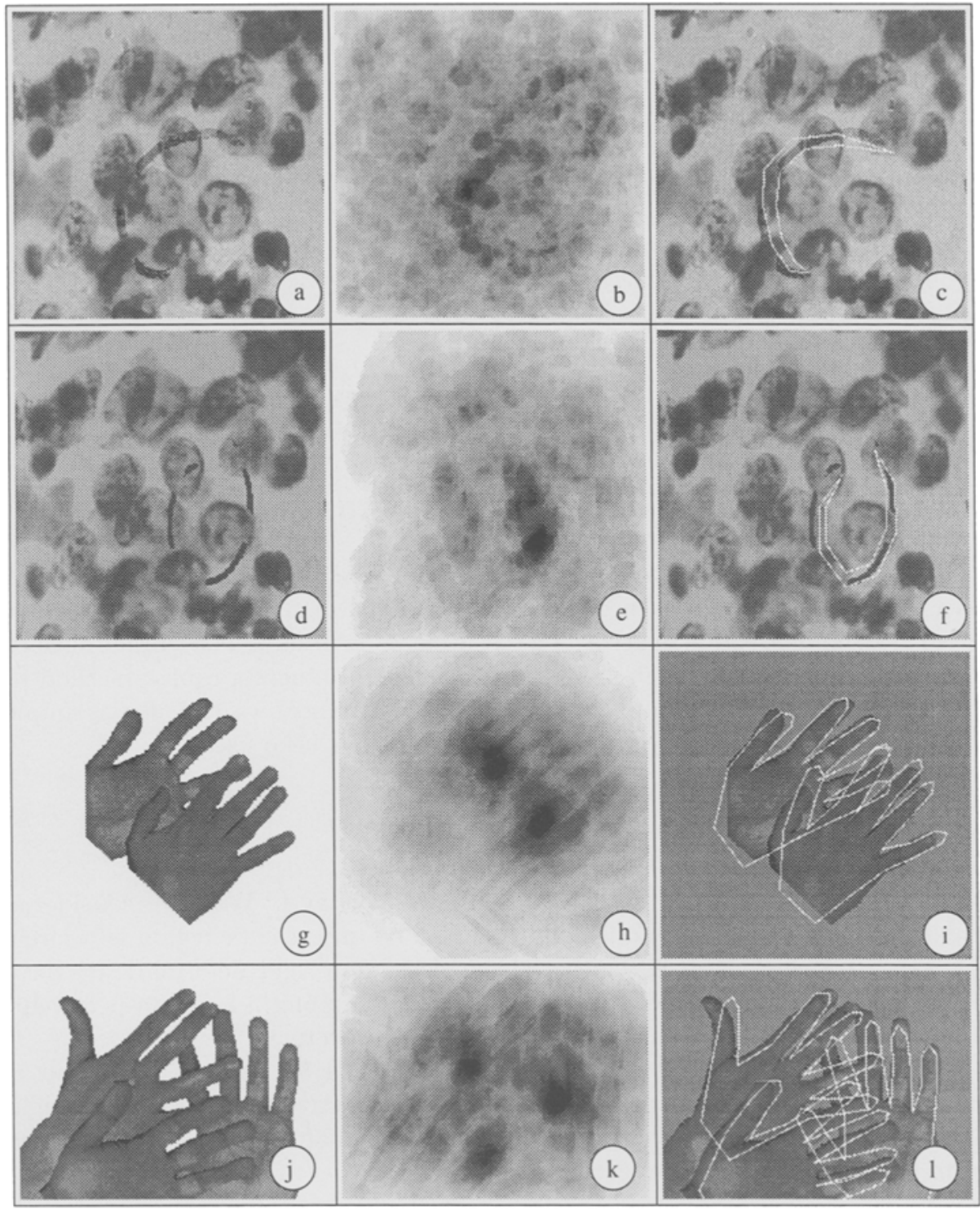

Fig. 1. Initialization.

in which the precision has been decreased to keep the required time and space under practical limits. Both, the initialization (using the reformulated H.T.) and optimization (using the proposed external energy function) method, make up a completely automatic algorithm independent of the object to be located, so this approach holds considerable promise not only as a technique to segment images but also, as a first work to design new and better algorithms. 


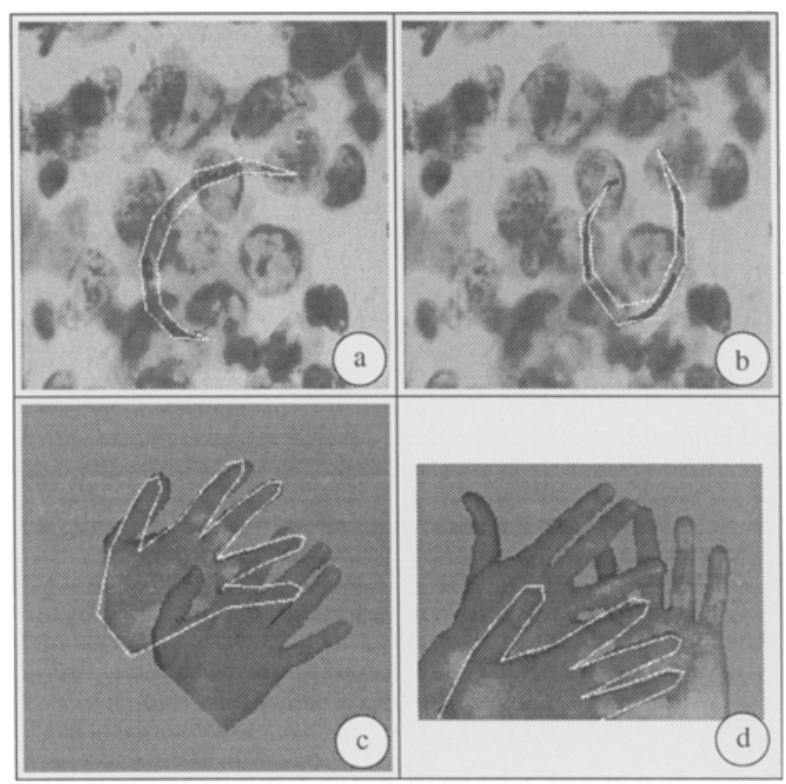

Fig. 2. Final results.

\section{References}

1. D.H. Ballard, Generalizing the Hough Transform to Detect Arbitrary Shapes, Pattern Recognition, vol. 13, pp. 111-122, 1981.

2. K. Bathe. Finite Element Procedures, Prentice-Hall, 1996.

3. J. Canny, A computacional approach to edge detection, IEEE Trans. PAMI, vol. 8, n. 6. pp 679-698, 1986.

4. L. D. Cohen and I. Cohen, Finite-Element Methods for Active Contour Models and Balloons for 2-D and 3-D Images, IEEE Trans. PAMI, vol. 15, no. 11, pp 1131-1147, 1993.

5. T.F. Cootes, C.J. Taylor, D.H. Cooper and J. Graham, Active shape models their training and application, Computer vision and image understanding, vol. $61(1), 38-59,1995$.

6. J. Illingworth, and J. Kittler, A Survey of the Hough transform, Comput. Vision, Graph \& Image Process., Vol 44, pp 87-116, 1988.

7. F. Leymarie and M.D. Levine, Tracking Deformable Objects in the Plane Using and Active Contour Model, IEEE Trans. PAMI, vol. 15, N. 6, pp. 617-634, June 1993.

8. A. Pentland and S. Sclaroff. Closed-form solutions for physically-based shape modeling and recognition. IEEE Trans. PAMI, 13(7), pp. 715-729, July 1991.

9. T. Poggio and V. Torre, Ill-posed Problems and Regularization Analysis in Early Vision, Proc. of AARPA Image Understanding Workshop, pp. 257-263, 1984.

10. S. Sclaroff and A. Pentland. Modal Matching for Correspondence and Recognition, IEEE Trans. PAMI, vol 17, no. 6, pp 545-561, 1995.

11. A.L. Yuille, P.W. Hallinan and D.S. Cohen, Feature Extraction from faces using deformables templates, Int. Jour. Computer Vision, vol. 8, no. 2, 99-111, 1992. 